
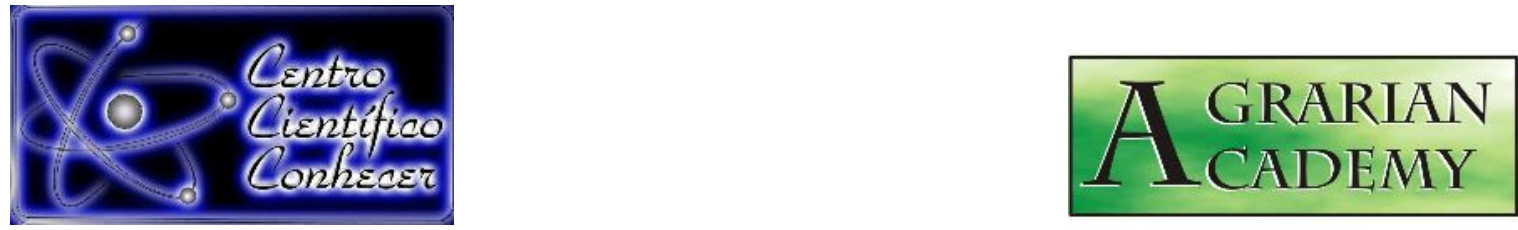

\title{
SUBSTRATOS NO CRESCIMENTO INICIAL DE MUDAS DE BARU
}

Stephany Diolino Cunha ${ }^{1}$, Sara Aparecida Guimarães de Souza ${ }^{2}$, Matheus da Silva Araújo $^{3}$, Karolayne Lemes D’Abadia ${ }^{1}$, Yago Cesar Rodrigues Morais ${ }^{4}$

1. Discentes do Curso de Engenharia Florestal da Universidade Estadual de Goiás, Campus Ipameri, Ipameri, GO, Brasil. (cunhaflorestal@outlook.com)

2. Discente do Curso de Agronomia do Centro Universitário de Anápolis -

UniEVANGÉLICA, Campus Anápolis, Anápolis, GO, Brasil.

3. Doutorando em Solos e Nutrição de Plantas da Universidade de São Paulo, Campus ESALQ, Piracicaba, SP, Brasil.

4. Engenheiro Florestal pela Universidade Estadual de Goiás, Campus Ipameri, Ipameri, GO, Brasil.

Recebido em: 19/11/2018 - Aprovado em: 14/12/2018 - Publicado em: 25/12/2018 DOI: 10.18677/Agrarian_Academy_2018B18

Objetivou-se avaliar o crescimento inicial de mudas de baru cultivadas em diferentes substratos. O experimento foi conduzido em casa de vegetação na Universidade Estadual de Goiás, Campus Ipameri, em sacos plásticos com capacidade de $8 \mathrm{dm}^{-3}$. $O$ delineamento experimental foi o inteiramente casualizado, com quatro tratamentos e cinco repetições. Os tratamentos foram constituídos de substratos formulados a base de: solo do cerrado (S1); solo do cerrado + esterco bovino curtido na proporção 2:1 (S2); solo do cerrado com saturação por bases corrigida para $60 \%$ (S3); e terriço de floresta (S4). As mudas foram conduzidas por 90 dias, época em que foram avaliadas as seguintes variáveis-respostas: altura, diâmetro do coleto, número de folíolos, área foliar, matéria seca da parte aérea, raiz, total e IQD. Os dados foram submetidos à análise de variância e teste de tukey. As mudas crescidas no S4 (terriço floresta) apresentaram as maiores médias em altura, diâmetro do coleto, e massa seca de folhas, caule, raiz e total, seguido do S3 (solo do cerrado com saturação por bases de $60 \%$ ), que também apresentaram mudas com maior crescimento em altura, diâmetro e massa seca. As mudas crescidas no S2 não diferiram daquelas crescidas com o $\mathrm{S} 1$. O maior crescimento das mudas observadas no S4 e S3 pode estar relacionado ao maior nível de fertilidade desses substratos. O uso de substrato formulado com terriço floresta propicia mudas de baru com maior qualidade para o campo.

PALAVRAS-CHAVE: Cerrado, fertilidade do solo, produção de mudas. 


\title{
SUBSTRATES ON GROWTH OF SEEDLINGS OF INICILAL BARU
}

\begin{abstract}
Objective to evaluate the initial growth of seedlings of cultivated in different substrata baru. The experiment was conducted in a greenhouse at the State University of Goiás, Ipameri, in plastic bags with a capacity of $8 \mathrm{dm}^{-3}$. The experimental design was completely randomized design with four treatments and five replications. The treatments were comprised of formulated the basis of substrates: cerrado soil (S1); cerrado soil + cow manure in the ratio 2:1 tanned (S2); cerrado soil with saturation by bases corrected to $60 \%$ (S3); and composts of forest (S4). The seedlings were conducted for 90 days, during which the following variables were evaluated-answers: height, diameter of the collect, number of leaflets, leaf area, dry weight of shoot, root, and total IQD. The data were subjected to analysis of variance and tukey test. Seedlings grown in S4 (composts forest) presented the highest average in height, diameter of the collect, and dry mass of leaves, stem, root and total, followed by the S3 (cerrado soil saturation of bases with $60 \%$ ), which also showed growing seedlings in height, diameter, and dry mass. Seedlings grown in S2 did not differ from those grown with the S1. The greatest seedling growth observed in the S4 and S3 can be related to the higher level of fertility of these substrates. The use of substrate formulated with composts forest provides seedlings of baru with higher quality to the field.
\end{abstract}

KEYWORDS: Cerrado, soil fertility, production of seedlings.

\section{INTRODUÇÃO}

É evidente que com o passar do tempo há uma maior preocupação com a degradação ambiental e perda de recursos naturais causados pelas pressões antrópicas sobre o meio ambiente. Esse fator influencia diretamente no interesse em atributos e estudos sobre as espécies florestais nativas, uma vez que a mesma representa e estabelece a grande riqueza gerada no nosso país. Uma ampla representação dessa diversidade denomina-se Cerrado, em que, esse bioma representa uma área de 203,4 milhões de hectares, o que totaliza aproximadamente $24 \%$ do território nacional (FIEDLER, 2008). Entretanto, para que não haja extinção desse bioma e de suas relevantes espécies nativas, faz-se necessário a realização de estudos com o intuito de estimular maior demanda de produção de mudas que consequentemente gerará significativa conservação desse ecossistema.

Desse modo, a espécie Dipteryx alata Vog. pertencente à família Fabaceae e popularmente conhecida como baru, cumaru ou cumbaru, é uma espécie arbórea do Cerrado, no qual, faz parte de um grupo de cerca de 110 espécies nativas que apresentam potencial econômico e está entre as 10 mais promissoras para cultivo, onde sua distribuição natural é bastante ampla, ocorrendo nos estados de Goiás, Minas Gerais, Mato Grosso do Sul, São Paulo e Distrito Federal (LEFB, 2014). É uma árvore que apresenta porte médio de 25 metros, com tronco chegando a $70 \mathrm{~cm}$ de diâmetro, exibindo consequentemente atributos relevantes, como: sombreamento de pastagens, recuperação de áreas degradada e também sua madeira, que é amplamente utilizada na construção civil, por ser considerada pesada e com alta resistência ao apodrecimento e ao ataque de organismos (VERA; SOUZA, 2009).

Devido a grande capacidade de adaptação, o baruzeiro exibe vasto potencial em sistemas produtivos e manifesta-se como uma espécie bastante interessante quando se trata do conforto térmico dos animais e alimento (SANO et al., 2004). No 
entanto, apesar de suas várias particularidades, poucos estudos são realizados quando se refere à produção de mudas e substratos adequados. Uma vez que ambos os processos realizados de forma pertinentes são extremamente importantes para o crescimento propício da mesma. Dessa forma, é indispensável que antes da implantação das mudas sejam realizados estudos referente ao desenvolvimento adequado, principalmente quando se refere ao tipo de substrato, em termos nutricionais e principalmente econômicos (BASÍLIO et al., 2018).

Sendo assim, a etapa de produção de mudas é a fase principal para obtenção da homogeneidade das plantas (COSTA et al., 2015). Nessa fase, há vários procedimentos que devem ser realizados a fim de proporcionar a obtenção de plantas com elevada qualidade, garantindo o sucesso no desenvolvimento a campo, como: o tipo de substrato, tipo de ambiente protegido, o volume de recipiente, a irrigação, a adubação e o manejo correto das operações de produção. Entretanto, para atingir a qualidade das mudas, é necessária a análise de parâmetros morfológicos e fisiológicos. Os aspectos morfológicos das mudas podem ser estabelecidos através da altura, diâmetro de colo, biomassa seca da parte aérea, das raízes e total (CARNEIRO, 1995).

Dentre os procedimentos citados para obtenção de mudas, o substrato se destaca e exerce papel fundamental no desempenho germinativo das sementes. Ele detém a função de manter as condições adequadas para germinação das mesmas e para o desenvolvimento das plântulas. Além de fornecer água, oxigênio e nutrientes, permitindo o crescimento das raízes e assim consequentemente oferecendo suporte estrutural à parte aérea (QUEIROZ; FIRMINO, 2014). No entanto, para escolher o tipo de substrato adequado, devem ser consideradas as características físicas e químicas, como: homogeneidade, alta porosidade, boa capacidade de retenção de água, boa agregação das partículas nas raízes, nutrientes em quantidades suficientes para o bom desenvolvimento das mudas, além de aspectos econômicos (SILVA; AGUIAR, 2004). Diante do exposto, este trabalho teve como objetivo avaliar o crescimento inicial de mudas de baru em diferentes substratos, visando à obtenção de mudas de qualidade.

\section{MATERIAL E MÉTODOS}

O experimento foi instalado e conduzido em casa de vegetação, na área experimental da Universidade Estadual de Goiás, Câmpus Ipameri (coordenadas

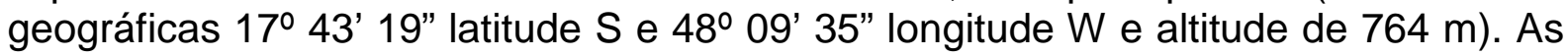
características da estufa utilizada são: $3,5 \mathrm{~m}$ de pé direito, 30,0 m comprimento, 7,0 $\mathrm{m}$ de largura, fechada nas laterais com sombrite $50 \%$ na cor preta e cobertura com plástico transparente 150 micras. O Município de Ipameri está localizado na região sudeste do Estado de Goiás, onde o clima é classificado como Aw (tropical estacional) com precipitação anual de aproximadamente $1600 \mathrm{~mm}$, sendo caracterizada por duas estações bem definidas, uma seca no inverno e uma chuvosa no verão, e com temperatura média de cerca de 23ํ $\mathrm{C}$ (ALVARES et al., 2013). As temperaturas de dentro da estufa foram da ordem de $10^{\circ} \mathrm{C}$ (mínima) e $44^{\circ} \mathrm{C}$ (máxima) e média de $29^{\circ} \mathrm{C}$.

As mudas de Dipteryx alata Vog. foram originadas por sementes coletadas no município de Orizona-GO e produzidas em sacos plásticos de dimensões de $8 \mathrm{dm}^{3}$, preenchidas com substratos oriundos das combinações de misturas de solo de cerrado (Latossolo Vermelho-Amarelo), esterco (curtido), terriço de floresta $(5 \mathrm{~cm}$ de profundidade) e NPK (4-14-8). A profundidade de semeadura foi de 1 a $3 \mathrm{~cm}$, sendo 
semeadas três sementes por recipiente. Posteriormente foi realizado o desbaste, deixando-se uma plântula em cada recipiente, iniciando-se o período experimental.

A análise físico-química, do solo de cerrado (Latossolo Vermelho-Amarelo distrófico) (EMBRAPA, 2013), apresentou os seguintes valores iniciais: 300, 80,0 e $620,0 \mathrm{~g} \mathrm{~kg}^{-1}$ de argila, silte e areia, respectivamente; $\mathrm{pH}\left(\mathrm{CaCl}_{2}\right)=5,1 ; \mathrm{H}+\mathrm{Al}=2,2$ $\mathrm{cmol}_{\mathrm{c}} \mathrm{dm}^{-3} ; \mathrm{Ca}=0,8 \mathrm{cmol}_{\mathrm{c}} \mathrm{dm}^{-3} ; \mathrm{Mg}=0,3 \mathrm{cmol}_{\mathrm{c}} \mathrm{dm}^{-3} ; \mathrm{P}$ (mehlich) =1,2 $\mathrm{mg} \mathrm{dm}^{-3} ; \mathrm{K}=$ $0,04 \mathrm{cmol}_{\mathrm{c}} \mathrm{dm}^{-3}$; Matéria orgânica $=9,0 \mathrm{~g} \mathrm{dm}^{-3} ; \mathrm{CTC}=3,36 \mathrm{cmol}_{\mathrm{c}} \mathrm{dm}^{-3} ; \mathrm{V} \%=34,5$; $\mathrm{Cu}=1,9 \mathrm{mg} \mathrm{dm}^{-3}, \mathrm{Fe}=43,9 \mathrm{mg} \mathrm{dm}^{-3}, \mathrm{Mn}=3,4 \mathrm{mg} \mathrm{dm}^{-3}, \mathrm{Zn}=0,2 \mathrm{mg} \mathrm{dm}^{-3}, \mathrm{~B}=0,19$ $\mathrm{mg} \mathrm{dm}^{-3}$, respectivamente. O substrato terriço de floresta apresentou os seguintes valores iniciais: $\mathrm{P}=2,70 \% ; \mathrm{K}=2,90 \% ; \mathrm{Ca}=4,03 \% ; \mathrm{Mg}=0,83 \% ; \mathrm{S}=0,05 \% ; \mathrm{B}=$ $81 \mathrm{mg} \mathrm{kg}^{-1} ; \mathrm{Cu}=35 \mathrm{mg} \mathrm{kg}^{-1} ; \mathrm{Fe}=19692 \mathrm{mg} \mathrm{kg}^{-1} ; \mathrm{Mn}=2409 \mathrm{mg} \mathrm{kg}^{-1} ; \mathrm{Zn}=90 \mathrm{mg} \mathrm{kg}^{-}$ 1; $\mathrm{Na}=6449 \mathrm{mg} \mathrm{kg}^{-1} ; \mathrm{CTC}=252 \mathrm{mmol} \mathrm{kg}^{-1}$.

O delineamento experimental utilizado foi o inteiramente casualizado, com quatro tratamentos e cinco repetições cada, totalizando 20 unidades (mudas) experimentais. Os tratamentos constituíram de 4 substratos formulados a base de: solo do cerrado (S1); solo do cerrado + esterco bovino curtido na proporção 2:1 (S2); solo do cerrado com saturação por bases corrigida para 60\% (S3); e terriço de floresta (S4), acondicionados em sacos plásticos com capacidade de $8 \mathrm{dm}^{3}$.

A umidade do solo foi mantida durante todo o período experimental em aproximadamente $60 \%$ da capacidade máxima de retenção de água do solo. 0 volume de água evapotranspirado foi reposto, diariamente, por meio de pesagem dos sacos, irrigados com água destilada.

Após 90 dias do desbaste, as características de crescimento foram avaliadas, por meio da medição de altura de plantas $(\mathrm{cm})(\mathrm{H})$, desde o colo até o ápice, com o auxílio de régua graduada, diâmetro do coleto $(\mathrm{mm})(\mathrm{DC})$, utilizando-se de um paquímetro digital e número de folíolos $\left(\mathrm{n}^{\circ}\right)(\mathrm{NF})$, por meio de contagem manual. Em seguida os folíolos das plantas foram escaneadas e com auxílio do programa Imaje, por diferença de cores foi calculada a área foliar.

Em seguida as plantas foram separadas em parte aérea e raízes para a determinação de massa seca. Realizou-se lavagem das partes das plantas com água destilada e, posteriormente, as mesmas foram colocadas em estufa de circulação forçada de ar por 72 horas, na temperatura de $70{ }^{\circ} \mathrm{C}$, até a obtenção de massa constante. Depois de secas, foram pesadas em balança analítica com precisão de $0,01 \mathrm{~g}$ para determinação da massa seca da parte aérea e raiz, cujos valores foram somados para obtenção da massa seca total (g) (MST).

O índice de qualidade de Dickson (IQD) foi determinado em função do peso de massa seca total (PMST), da altura da parte aérea $(H)$, do diâmetro do coleto (DC), do peso de massa seca da parte aérea (PMSPA) e do peso da massa seca das raízes (PMSR), por meio da fórmula (DICKSON et al., 1960);

$$
\frac{\operatorname{PMST}(\mathrm{g})}{\frac{\mathrm{H}(\mathrm{cm})}{\mathrm{DC}(\mathrm{mm})}+\operatorname{PMSPA}(\mathrm{g}) / \operatorname{PMSR}(\mathrm{g})}
$$

Com os dados obtidos, foi realizada à análise de variância aplicando-se o teste $F$ para significância, com $\alpha=0,05$. Testaram-se os pressupostos de normalidade e homocedasticidade dos resíduos. Por meio do teste de Tukey $(\alpha=0,05)$, compararam-se as médias dos tratamentos.

AGRARIAN ACADEMY, Centro Científico Conhecer - Goiânia, v.5, n.10; p. 1942018 
As análises estatísticas foram efetuadas utilizando os softwares $R$ versão 3,2 (R DEVELOPMENT CORE TEAM, 2015), e o pacote Vegan (OKSANEN et al., 2016).

\section{RESULTADOS E DISCUSSÃO}

Para as variáveis analisadas (altura, diâmetro do coleto, número de folíolos e área foliar) verifica-se que houve diferença significativa pelo Teste de $\mathrm{F}$ entre os tratamentos (Tabela 1). No qual, as mudas desenvolvidas no tratamento S4 (terriço de floresta) apresentaram as maiores médias nas variáveis analisadas, seguido do S3 (solo do cerrado com saturação por bases a 60\%), que também apresentaram plantas com maior crescimento em altura, diâmetro do coleto, número de folíolos e área foliar. Tal resultado está diretamente relacionado com o substrato terriço de floresta, os substratos advindos de várzea são formados pela decomposição de substâncias animais e vegetais, substratos assim apresentam teores satisfatórios de matéria orgânica, níveis adequados de fertilidade, alta porosidade e boa retenção de umidade, condições que são essenciais para o desenvolvimento inicial das mudas (CRUZ et al., 2013).

TABELA 1. Médias de porcentagem de altura de plantas $(H)$, diâmetro do coleto (DC), número de folíolos (NF) e área foliar (AF) de plantas de baru em diferentes substratos.

\begin{tabular}{ccccc}
\hline Substratos & $\begin{array}{c}\mathrm{H} \\
(\mathrm{cm})\end{array}$ & $\begin{array}{c}\mathrm{DC} \\
(\mathrm{mm})\end{array}$ & $\begin{array}{c}\mathrm{NF} \\
\left(\mathrm{n}^{\circ}\right)\end{array}$ & $\begin{array}{c}\mathrm{AF} \\
\left(\mathrm{mm}^{2}\right)\end{array}$ \\
\hline $\mathrm{S} 1$ & $12.4 \mathrm{~b}$ & $6.4 \mathrm{~b}$ & $30 \mathrm{c}$ & $153.5 \mathrm{~b}$ \\
$\mathrm{~S} 2$ & $19.8 \mathrm{ab}$ & $8.4 \mathrm{ab}$ & $49 \mathrm{~b}$ & $169.7 \mathrm{~b}$ \\
$\mathrm{~S} 3$ & $26.2 \mathrm{a}$ & $8.6 \mathrm{ab}$ & $50 \mathrm{~b}$ & $591.2 \mathrm{a}$ \\
$\mathrm{S} 4$ & $27.0 \mathrm{a}$ & $10.0 \mathrm{a}$ & $70 \mathrm{a}$ & $604.1 \mathrm{a}$ \\
\hline $\mathrm{CV}(\%)$ & 22.6 & 16.8 & 14.0 & 19.2 \\
\hline Teste $\mathrm{F}$ & $9.86^{* *}$ & $5.56^{* *}$ & $27.5^{* *}$ & $59.2^{* *}$ \\
\hline
\end{tabular}

**Significativo ao nível de $1 \%$ de probabilidade pelo Teste F. Médias seguidas de mesma letra minúscula na coluna, não diferem entre si pelo teste de tukey a $5 \%$ de probabilidade. S1 (solo do cerrado); S2 (solo do cerrado + esterco bovino curtido na proporção 2:1); S3 (solo do cerrado com saturação por bases corrigida para $60 \%$ ); e (S4) terriço de floresta.

As mudas do tratamento S2 (solo de cerrado + esterco bovino curtido na proporção de 2:1), não diferiram das crescidas no $\mathrm{S1}$ (solo de cerrado). No qual, esse evento pode resultar futuramente em uma menor resistência às condições adversas impostas pelos fatores ambientais (GOMES et al., 2002). Sendo que, segundo Delarmelina et al. (2014) o substrato tem grande influência no processo de formação de mudas de qualidade, principalmente nas fases iniciais da vida das plantas.

Sendo assim, os menores valores (altura, diâmetro do coleto, número de folíolos e área foliar) do tratamento $S 1$ e $S 2$ podem estar relacionados ao menor nível de fertilidade desses substratos. Porém, vale ressaltar que esses substratos não apresentaram mortalidade $e$ danos às mudas. Contudo, 0 efeito significativamente positivo dos substratos S3 e S4 no crescimento em altura de mudas podem estar relacionados com a maior disponibilidade de nutrientes desses substratos, favorecendo o crescimento das mudas, no qual, esse pôde ter 
influenciado em uma maior retenção e disponibilidade de água para manter a turgescência e metabolismo da parte aérea (BONAMINGO et al., 2016).

Em relação ao número de folíolos e área foliar, é nítida a discrepância do substrato $\mathrm{S4}$, cujo resultado foi significativamente maior em relação aos demais. É importante salientar que as mudas com maior área foliar e número de folhas tendem a aumentar sua taxa fotossintética, favorecendo assim o crescimento. Dessa forma, analisando os dados do tratamento mencionado, é notório o grande potencial da utilização desse substrato para a produção de mudas, representado pelos efeitos favoráveis quando comparados aos outros substratos. Da mesma forma, quando compara a altura do substrato $\mathrm{S} 4$, nota-se que esse apresentou maior comprimento. Esta característica, do comprimento das plantas, bem como diâmetro do colo e número de folhas das mesmas deve ser observada com cautela, afinal, a altura da parte aérea se correlaciona positivamente com a biomassa (SILVA FILHO et al., 1995).

O desenvolvimento em relação a altura e diâmetro do colo constitui um dos parâmetros usados para avaliar a qualidade de mudas florestais, pois, além de refletir o acúmulo de reservas, proporciona também maior resistência e melhor fixação no solo. Essa variável é reconhecida como um dos melhores indicadores do padrão de qualidade de mudas, sendo, em geral, o mais indicado para determinar a capacidade de sobrevivência de mudas no campo (ARAUJO et al., 2018).

Analisando as médias da massa seca parte aérea, raiz, total e o índice de qualidade de Dickson observa-se que os melhores resultados foram das plantas submetidas ao tratamento S4, seguido dos tratamentos S2 e S3 que não diferiram entre si e por fim o tratamento S1 no qual apresentou menor média (Tabela 2). É importante destacar que o tratamento $\mathrm{S} 1$ é composto somente por solo de cerrado, sendo um solo naturalmente ácido e de baixa fertilidade (CABRAL et al., 2016), fato que pode explicar o baixo crescimento das plantas submetidas a este tratamento.

TABELA 2. Médias de porcentagem de massa seca parte aérea (MSPA), massa seca de raiz (MSR), massa seca total (MST) e índice de qualidade de Dickson (IQD) de plantas de baru em diferentes substratos.

\begin{tabular}{ccccc}
\hline Substratos & MSPA & MSR & MST & IQD \\
\hline S1 & $(\mathrm{g})$ & $(\mathrm{g})$ & $(\mathrm{g})$ & $2.5 \mathrm{c}$ \\
S2 & $4.6 \mathrm{c}$ & $3.6 \mathrm{c}$ & $8.2 \mathrm{c}$ & $5.1 \mathrm{~b}$ \\
S3 & $16.6 \mathrm{~b}$ & $8.2 \mathrm{~b}$ & $25.6 \mathrm{~b}$ & $6.1 \mathrm{~b}$ \\
S4 & $20.4 \mathrm{~b}$ & $9.2 \mathrm{~b}$ & $28.6 \mathrm{~b}$ & $9.3 \mathrm{a}$ \\
\hline CV (\%) & $34.4 \mathrm{a}$ & $13.8 \mathrm{a}$ & $48.4 \mathrm{a}$ & 15.3 \\
\hline Teste F & 19.4 & 12.7 & 11.9 & $49.1^{\star \star}$ \\
\hline
\end{tabular}

**Significativo ao nível de $1 \%$ de probabilidade pelo Teste F. Médias seguidas de mesma letra minúscula na coluna, não diferem entre si pelo teste de tukey a $5 \%$ de probabilidade. S1 (solo do cerrado); S2 (solo do cerrado + esterco bovino curtido na proporção 2:1); S3 (solo do cerrado com saturação por bases corrigida para $60 \%$ ); e (S4) terriço de floresta.

O maior índice de qualidade de Dickson $(9,3)$ foi obtido com a utilização do substrato S4 (Tabela 2), indicando a importância da escolha de um bom substrato para o crescimento equilibrado do baru em fase inicial de desenvolvimento. $O$ índice é uma ferramenta fundamental na avaliação de qualidade de mudas, pois se leva em

AGRARIAN ACADEMY, Centro Científico Conhecer - Goiânia, v.5, n.10; p. 1962018 
consideração o equilíbrio da distribuição da biomassa das plantas, assim quanto maior o índice, maior será a capacidade das mudas sobreviverem e se estabelecerem no campo (ELOY et al., 2013; SILVA et al., 2013; SOUZA et al., 2013).

Por fim, o maior crescimento das mudas no substrato com terriço de floresta seguido do solo do cerrado com saturação por bases corrigida para $60 \%$ indica que estes compostos foram essenciais para aumentar a qualidade das mudas. A presença desses, possivelmente, melhorou as características de aeração, estrutura e retenção de água, teor de matéria orgânica e quantidade de nutrientes, permitindo melhor desenvolvimento das mudas.

\section{CONCLUSÃO}

O uso de substrato formulado com terriço floresta propicia mudas de baru com maior qualidade para o campo.

\section{REFERÊNCIAS}

ALVARES, C. A.; STAPE, J. L.; SENTELHAS, P. C.; GONÇALVES, M. L. M.; SPAROVEK, G. Köppen's climate classification map for Brazil. Meteorologische Zeitschrift v. 22, n. 6, p. 711-728, 2013. Disponível em: < https://www.schweizerbart.de/papers/metz/detail/22/82078/Koppen_s_climate_classif ication_map_for_Brazil?af=crossref $>$. doi: 10.1127/0941-2948/2013/0507

ARAUJO, M. S.; D'ABADIA, K. L.; CUNHA, S. D.; COELHO, G. M. MORAIS, Y. C. R.; BARRETTO, V. C. M.; CALIXTO JUNIOR, J. E. D. Efeitos da fertilização potássica no crescimento inicial de Khaya senegalensis A. Juss no bioma Cerrado. Ecologia e Nutrição Florestal, v. 6, n. 1, p. 8-16, 2018. Disponível em: < http://dx.doi.org/10.5902/2316980X26186>. doi: 10.5902/2316980X26186

BAsílio, A. C. A.; BARBOSA, G. D.; COELHO, G. M.; ARAUJO, M. A.; ZUCCHI, M. R. Emergência e desenvolvimento de plântulas de Dipteryx alata vogel em diferentes substratos. Revista Agrotecnologia, v. 9, n. 2, p. 57-65, 2018. Disponível em: $<$ http://dx.doi.org/10.12971/2179-5959/agrotecnologia.v9n2p57-65>. doi: 10.12971/2179-5959/agrotecnologia.v9n2p57-65

BONAMIGO, T.; SCALON, S. P. Q.; PEREIRA, Z. V.; Substratos e níveis de luminosidade no crescimento inicial de mudas de Tocoyena formosa (Cham. E Schltdl.) K. Schum. (RUBIACEAE). Ciência Florestal, v. 26, n. 2, p. 501-511, 2016. Disponível em: <http://dx.doi.org/10.5902/1980509822750>. doi: $10.5902 / 1980509822750$

CABRAL, C. E. A.; CABRAL, L. S.; SILVA, E. M. B.; CARVALHO, K. S. C.; KROTH, B. E.; CABRAL, C. H. A. Resposta da Brachiaria brizantha cv. Marandu a fertilizantes nitrogenados associados ao fosfato natural reativo. Comunicata Scientiae, Piaui, v.7, n.1, p.66-72, 2016.

CARNEIRO, J. G. A. Produção e controle de qualidade de mudas florestais. Curitiba: UFPR/FUPEF; 1995. 451 p. 
COSTA, E.; DIAS, J. G.; LOPES, K. G.; BINOTTI, F. F. S.; CARDOSO, E. D. Telas de Sombreamento e Substratos na Produção de Mudas de Dipteryx alata Vog. Floresta e Ambiente, v. 22, n. 3, p. 416-425, 2015. Disponível em: < http://dx.doi.org/10.1590/2179-8087.071714>. doi: 10.1590/2179-8087.071714

CRUZ, F. R. S.; ALVES, E. U.; SILVA, R. S.; ANDRADE, L. A.; ARAUJO, L. R. Emergência e crescimento inicial de plântulas de Enterolobium contortisiliquum (Vell.) Morong em diferentes substratos. Scientia Plena, v. 9, n. 12, p. 1-9, 2013. Disponível em: < https://www.scientiaplena.org.br/sp/article/view/1505/925>.

DELARMELINA, W. M.; CALDEIRA, M. V. W.; FARIA, J. C. T.; GONÇALVES, E. O.; ROCHA, R. L. F. Diferentes Substratos para a Produção de Mudas de Sesbania virgata. Floram, v. 21, n. 2, p. 224-233, 2014. Disponível em: < http://dx.doi.org/10.4322/floram.2014.027>. doi: 10.4322/floram.2014.027

DICKSON, A.; LEAF, A. L.; HOSNER, J. F. Quality appraisal of white spruce and white pine seedling stock in nurseries. Forestry Chronicle, v. 36, n. 1, p. 10-13, 1960. Disponível em: <https://doi.org/10.5558/tfc36010-1>. doi: doi.org/10.5558/tfc36010-1

ELOY, E.; CARON, B. O.; SCHMIDT, D.; BEHLING, A.; SCHWERS, L.; ELLI, E. F. Avaliação da qualidade de mudas de Eucalyptus grandis utilizando parâmetros morfológicos. Floresta, v. 43, n. 3, p. 373-384, 2013. Disponível em: <http://dx.doi.org/10.5380/rf.v43i3.26809>. doi: 10.5380/rf.v43i3.26809

EMBRAPA. Sistema brasileiro de classificação de solos. 3 ed. Brasília: Embrapa Solos, 2013. 201p.

FIEDLER, N. C.; SOARES, T. S.; SILVA, G. F. Produtos Florestais Não Madeireiros: Importância e Manejo Sustentável da Floresta. Revista Ciências Exatas e Naturais, v. $10, \quad$ n. 2, p. 263-268, 2008. Disponível em: < https://revistas.unicentro.br/index.php/RECEN/article/view/712/885>.

GOMES, J. M.; COUTO, L.; LEITE, H. G.; XAVIER, A.; GARCIA, S. L. R. Parâmetros morfológicos na avaliação da qualidade de mudas de Eucalyptus grandis. Revista Árvore, v. 26, n. 6, p. 655-664, 2002. Disponível em: <http://www.scielo.br/pdf/rarv/v26n6/a02v26n6.pdf>.

LISTA DE ESPÉCIES DA FLORA DO BRASIL. Jardim Botânico do Rio de Janeiro, 2014. Disponível em: <http://floradobrasil.jbrj.gov.br/>. Acesso em: 15 Ago. 2018.

OKSANEN, J.; F. BLANCHET, G.; FRIENDLY, M.; KINDT, R.; LEGENDRE, P.; MCGLINN, D.; PETER R. M.; O'HARA, R. B.; GAVIN, L. S.; PETER, S. M.; STEVENS, H. H.; SZOECS, E.; WAGNER, H. Vegan: community ecology package. R package version 2.4-0. Available at. 2016. Acessado em 15 mar. 2017. Online. Disponível em: https://CRAN.Rproject.org/package=vegan. 
QUEIROZ, S. E. E.; FIRMINO, T. O. Efeito do sombreamento na germinação e desenvolvimento de mudas de baru (Dipteryx alata Vog.). Revista Biociências, v. 20, n. 1, p. 72-77, 2014. Disponível em: < http://periodicos.unitau.br/ojs/index.php/biociencias/article/view/1837/1520>.

R CORE TEAM. 2017. R: A Language and Environment for Statistical Computing. Vienna, Austria, R Foundation for Statistical Computing. Available from: https://www.R-project.org/. Accessed: Nov. 17, 2017.

SANO S. M, RIBEIRO J. F, BRITO M. A. Baru: biologia e uso [online]. Planaltina: Embrapa Cerrados; 2004. Documentos n. 116. Disponível em: www. cpac.embrapa.br/download/336/t.

SILVA FILHO.; NODA, H.; CLEMENT, C. R.; MACHADO, F. M. Efeito da adubação orgânica na produção de biomassa em quebra-pedra (Phyllanthus stipulatus, Euphorbiaceae) em Manaus, Brasil. Acta Amazônica, v. 27, n. 2, p. 73-80, 1995. Disponível em: <http://dx.doi.org/10.1590/1809-43921997272080>. doi: $10.1590 / 1809-43921997272080$

SILVA, P. M. C. S.; UCHOA, S. BARBOSA, J. B. F.; BASTOS, V. J.; ALVES, J. M. A.; FARIAS, L. C. Efeito do potássio e do calcário na qualidade de mudas de cedro doce (Bombacopsis quinata). Revista Agro@mbiente On-line, v. 7, n. 1, p. 63-69, 2013. Disponível em: < https://revista.ufrr.br/agroambiente/article/view/842/1022>.

SILVA, L. M. M.; AGUIAR, I. B. Efeito dos substratos e temperaturas na germinação de sementes de Cnidosculus phyllacanthus Pax e K. Hoffm. (Faveleira). Revista Brasileira de Sementes, v. 26, n. 1, p. 9-14, 2004. Disponível em: < http://dx.doi.org/10.1590/S0101-31222004000100002>. doi: 10.1590/S010131222004000100002

SOUZA, N. H.; MARCHETTI, M. E.; CARNEVALI, T. O.; RAMOS, D. D.; SCALON, S. P. Q.; SILVA, E. F. Estudo nutricional da canafístula (I): crescimento e qualidade de mudas em resposta à adubação com nitrogênio e fósforo. Revista Árvore, v. 37, n. 4, p. 717-724, 2013. Disponível em: < http://www.redalyc.org/html/488/48828747015/>.

VERA, R.; SOUZA E. R. B. Baru. Revista Brasileira de Fruticultura. v. 31, n. 1, p. 1-295, 2009. Disponível em: <http://dx.doi.org/10.1590/S010029452009000100001>. doi: 10.1590/S010029452009000100001 